\title{
Hölder stability in Type III thermoelastodynamics
}

\author{
M.C. Leseduarte and R. Quintanilla
}

\author{
Matemàtica Aplicada 2, ETSEIAT, Universitat Politècnica de Catalunya \\ Colom, 11. Terrassa (08222). Barcelona. Spain \\ E-mail addresses: Mari.Carme.Leseduarte@upc.edu, Ramon.Quintanilla@upc.edu
}

To G.A. Maugin in his 70th birthday

\begin{abstract}
This note is concerned with the linear (and linearized) Type III thermoelastodynamic theory proposed by Green and Naghdi. We here assume that the mass density is positive and the thermal conductivity tensor is positive definite. However, we do not assume the positivity of any other tensor. In this situation, we obtain Hölder continuous dependence results on the supply terms. We also sketch how to prove the continuous dependence on the initial data.
\end{abstract}

keywords: Type III thermoelastodynamics, Hölder stability, Continuous dependence on initial data and supply terms, Lagrange identities method

\section{Introduction}

The thermoelastic theory proposed by Green and Naghdi [5-7] has deserved an intense investigation in the last years. Three sub-theories, where an entropy balance law replaces the customary entropy inequality, have been proposed. These theories were labelled as Type I, II and III. The linear version of Type I agrees with the usual classical theory of thermoelasticity. For Type II, the energy of the system is constant with respect to the time. For this reason it is also known as "thermoelasticity without energy dissipation". Type III is the more general theory and it contains the other two as limiting cases. We can recall several papers (see Iesan [8,9]; Iesan and Quintanilla [10]; Lazzari and Nibbi [13]; Leseduarte et al. [14,15]; Liu and Quintanilla [16, 17]; Liu and Lin [18]; Messaoudi and Soufyane [19]; Puri and Jordan [20]; Qin et al. [21]; Quintanilla [22-27]; Quintanilla and Racke [28]; Quintanilla and Straughan [29-31]; Yang and Wang [34], among others), where existence, uniqueness, continuous dependence, spatial and time behavior have been studied.

We recall that the linear system for the centrosymmetric Type III thermoelastodyamics can be written as

$$
\left\{\begin{aligned}
\rho \ddot{u}_{i} & =\left(a_{i j k h} u_{k, h}-a_{i j} \theta\right)_{, j}+\rho f_{i}, \\
c \dot{\theta} & =-a_{i j} \dot{u}_{i, j}+\left(k_{i j} \theta_{, i}\right)_{, j}+\left(b_{i j} \alpha_{, i}\right)_{, j}+\rho r,
\end{aligned}\right.
$$

where $u_{i}$ is the displacement vector, $\theta$ is the temperature, $\rho$ is the mass density, $c$ is the thermal capacity, $\left(a_{i j k h}\right)$ is the elasticity tensor, $a_{i j}$ is the coupling tensor, $k_{i j}$ is the thermal 
conductivity tensor and $b_{i j}$ denotes a tensor which is typical for the Types II and III theories. The constitutive tensors $\rho, c, a_{i j k h}, a_{i j}, b_{i j}$ and $k_{i j}$ are smooth functions of the position. They satisfy the symmetries

$$
a_{i j k h}=a_{k h i j}
$$

and

$$
k_{i j}=k_{j i}, b_{i j}=b_{j i}
$$

The thermal displacement $\alpha$ is defined by

$$
\alpha(\boldsymbol{x}, t)=\int_{0}^{t} \theta(\boldsymbol{x}, s) d s+\alpha_{0}(\boldsymbol{x})
$$

and $f_{i}$ and $r$ are the supply terms. To guarantee the well-posedness of the Type III thermoelasticity, we need to assume that the mass density and the heat capacity are positive as well as the elasticity tensor and the thermal conductivity. Lyapunov stability of the solutions is implied when $b_{i j}$ is also positive definite.

In the case where we do not assume the positivity of the elasticity tensor $a_{i j k h}$, the problem determined by the system (1.1) with usual initial and boundary conditions becomes ill-posed. It is worth recalling that this condition can be present in the case of prestressed solids.

It is worth noting several references for this situation. For instance, results concerning uniqueness and growth of solutions have been obtained at $[25,29]$ under the condition that the tensors $k_{i j}$ and $b_{i j}$ are positive. It is also worth mentioning that the only contribution concerning the case when $a_{i j k h}$ and $b_{i j}$ are not definite was obtained recently (see [15]). There, it was showed the uniqueness of solutions. We here want to show how to obtain the continuous dependence with respect to the supply terms and initial data under similar restrictions.

In 1960, John [11] showed how to obtain continuous dependence results in the sense of Hölder. This concept is weaker than the usual definition of the continuous dependence. The basic idea consists to impose that the solutions belong to a suitable constraint class. Since this contribution, many investigations have been directed to this kind of results. We may cite the works of Ames and Payne [1] and Knops and Payne [12] concerning isothermal elastodynamics. We can also recall that Hölder stability results can be found in different frameworks from the thermoelasticity (see Cimmelli and dell'Isola [3] and dell'Isola [4]). For the classical thermoelastodynamics, we may recall the contributions of Wilkes [33], Ames and Straughan [2] and Rionero and Chirita [32]. It is worth recalling and comparing several related results. In [15], we proposed a uniqueness result under similar assumptions to the ones considered here and in [24] the author proposed a Hölder stability result by using the logarithmic convexity argument under the assumption that $b_{i j}$ is positive definite. We here obtain a new result proving the Hölder stability of the solutions when we do not assume that the elasticity tensor neither the $b_{i j}$ tensor are positive definite. It is known that two usual techniques to study ill-posed problems in thermoelasticity are the logarithmic convexity and the Lagrange identities method. We here consider the second one. We want to emphasize that since the tensor $b_{i j}$ is not positive definite, it does not seem possible to apply the logarithmic convexity arguments. However, we here are able to use the Lagrange identities method. In fact, Lagrange identity method allows us to obtain equality (3.5) that implies the inequality (3.12). This inequality is the starting point to develop our approach. Therefore, Lagrange identity is the fundamental ingredient to obtain our results. 
The plain of this note is the following. In the next section we recall the basic assumptions and the conditions defining the problem. A basic inequality is obtained in Section 3. Hölder's continuous dependence with respect to the supply terms is obtained in Section 4. In the last section we sketch how to extend the argument to prove continuous dependence with respect to the initial data.

\section{Preliminaries}

In this section we propose the basic assumptions where we are going to work with. We study smooth solutions of (1.1) on $B \times I$, where $I$ is a bounded time interval and $B$ is a regular domain with boundary $\Gamma$ smooth enough to apply the Divergence Theorem.

In what follows we suppose that the constitutive tensors are bounded about and have the following properties:

(A1) The mass density $\rho$ and the heat capacity $c$ are positive functions. That is,

$$
\rho(\boldsymbol{x}) \geq \rho_{0}>0, \quad c(\boldsymbol{x}) \geq c_{0}>0, \quad \boldsymbol{x} \in B .
$$

(A2) The thermal conductivity tensor $k_{i j}$ is positive definite. That is, there exists a positive constant $C$ such that

$$
k_{i j} \xi_{i} \xi_{j} \geq C \xi_{i} \xi_{i}
$$

for every vector $\left(\xi_{i}\right)$.

The physical meaning of condition (A1) is obvious. Condition (A2) guarantees that the dissipation of the system is not negative and then, the energy does not increase. It is an usual assumption in the thermomechanical studies. We also note that from (2.1), the following inequality

$$
\left|b_{i j} \xi_{i} \xi_{j}\right| \leq C_{1} k_{i j} \xi_{i} \xi_{j}
$$

is satisfied for every vector $\left(\xi_{i}\right)$, where $C_{1}$ is a calculable constant ${ }^{1}$ which depends on the tensors $k_{i j}$ and $b_{i j}$.

To the field equations we adjoin the boundary conditions

$$
u_{i}(\boldsymbol{x}, t)=\bar{u}_{i}(\boldsymbol{x}, t), \alpha(\boldsymbol{x}, t)=\bar{\alpha}(\boldsymbol{x}, t), \boldsymbol{x} \in \Gamma, t \in I,
$$

together with the initial conditions

$$
u_{i}(\boldsymbol{x}, 0)=u_{i}^{0}(\boldsymbol{x}), \dot{u}_{i}(\boldsymbol{x}, 0)=v_{i}^{0}(\boldsymbol{x}), \alpha(\boldsymbol{x}, 0)=\alpha^{0}(\boldsymbol{x}), \dot{\alpha}(\boldsymbol{x}, 0)=\theta^{0}(\boldsymbol{x}), \boldsymbol{x} \in B .
$$

To study the continuous dependence of the solutions with respect to the supply terms, we denote by $\left(u_{i}^{(1)}, \alpha^{(1)}\right)$ the solution corresponding to the external data $\left(f_{i}^{(1)}, r^{(1)}\right)$ and by $\left(u_{i}^{(2)}, \alpha^{(2)}\right)$, the solution corresponding to the supply terms $\left(f_{i}^{(2)}, r^{(2)}\right)$.

We introduce the notation

$$
u_{i}=u_{i}^{(2)}-u_{i}^{(1)}, \quad \alpha=\alpha^{(2)}-\alpha^{(1)}, \quad F_{i}=f_{i}^{(2)}-f_{i}^{(1)}, \quad q=r^{(2)}-r^{(1)} .
$$

\footnotetext{
${ }^{1}$ We note that $C_{1}=b^{*} / k_{*}$, where $b^{*}$ is the maximum of the absolute values of the eigenvalues of the matrix $b_{i j}$ and $k_{*}$ is the minimum of the eigenvalues of $k_{i j}$.
} 
It follows that $\left(u_{i}, \alpha\right)$ satisfies the problem determined by the system

$$
\left\{\begin{aligned}
\rho \ddot{u}_{i} & =\left(a_{i j k h} u_{k, h}-a_{i j} \theta\right)_{, j}+\rho F_{i}, \\
c \dot{\theta} & =-a_{i j} \dot{u}_{i, j}+\left(k_{i j} \theta_{, i}\right)_{, j}+\left(b_{i j} \alpha_{, i}\right)_{, j}+\rho q,
\end{aligned}\right.
$$

with the homogeneous boundary conditions

$$
u_{i}(\boldsymbol{x}, t)=\alpha(\boldsymbol{x}, t)=0, \boldsymbol{x} \in \Gamma, t \in I,
$$

and the null initial conditions

$$
u_{i}(\boldsymbol{x}, 0)=\dot{u}_{i}(\boldsymbol{x}, 0)=\alpha(\boldsymbol{x}, 0)=\dot{\alpha}(\boldsymbol{x}, 0)=0, \boldsymbol{x} \in B .
$$

In our studies it will be useful the following inequality

$$
\int_{0}^{t} k_{i j} \alpha_{, i} \alpha_{, j} d s \leq \frac{4 C_{2}^{2} t^{2}}{\pi^{2}} \int_{0}^{t} k_{i j} \dot{\alpha}_{, i} \dot{\alpha}_{, j} d s
$$

which is satisfied for every function $\alpha$ such that $\alpha(0)=0$ and where $C_{2}$ is a calculable constant ${ }^{2}$.

\section{A basic inequality}

In this section we obtain an inequality satisfied by the solutions of the problem defined by the system (2.6) with boundary and initial conditions (2.7) and (2.8), respectively. This inequality will be relevant to obtain our results.

As we assume null Dirichlet boundary conditions and null initial conditions, we obtain that the energy equality

$$
\begin{aligned}
& \int_{B}\left(\rho \dot{u}_{i} \dot{u}_{i}+c \theta^{2}+a_{i j k h} u_{i, j} u_{k, h}+b_{i j} \alpha_{, i} \alpha_{, j}\right) d v \\
& \quad+2 \int_{0}^{t} \int_{B} k_{i j} \theta_{, i} \theta_{, j} d v d s-2 \int_{0}^{t} \int_{B}\left(\rho F_{i} \dot{u}_{i}+\rho q \theta\right) d v d s=0
\end{aligned}
$$

is satisfied for every solution of our problem.

On the other hand, from the equalities

$$
\begin{gathered}
\frac{d}{d s}\left[\rho \dot{u}_{i}(s) \dot{u}_{i}(2 t-s)\right]=\rho \ddot{u}_{i}(s) \dot{u}_{i}(2 t-s)-\rho \dot{u}_{i}(s) \ddot{u}_{i}(2 t-s), \\
\frac{d}{d s}[c \theta(s) \theta(2 t-s)]=c \dot{\theta}(s) \theta(2 t-s)-c \theta(s) \dot{\theta}(2 t-s)
\end{gathered}
$$

and the null initial and boundary conditions, we obtain

$$
\begin{aligned}
& \int_{B}\left(\rho \dot{u}_{i} \dot{u}_{i}+b_{i j} \alpha_{, i} \alpha_{, j}-a_{i j k h} u_{i, j} u_{k, h}-c \theta^{2}\right) d v \\
& =\int_{0}^{t} \int_{B} \rho\left(F_{i}(s) \dot{u}_{i}(2 t-s)-F_{i}(2 t-s) \dot{u}_{i}(s)\right) d v d s \\
& \quad-\int_{0}^{t} \int_{B} \rho(q(s) \theta(2 t-s)-q(2 t-s) \theta(s)) d v d s .
\end{aligned}
$$

\footnotetext{
${ }^{2}$ We note that $C_{2}^{2}=k^{*} / k_{*}$, where $k^{*}$ is the maximum of the eigenvalues of the symmetric matrix $k_{i j}$ and $k_{*}$ is the minimum of the eigenvalues of $k_{i j}$.
} 
From the equality (3.1) and the relation (3.4), we conclude that

$$
\begin{aligned}
& \int_{B}\left(\rho \dot{u}_{i} \dot{u}_{i}+b_{i j} \alpha_{, i} \alpha_{, j}\right) d v+\int_{0}^{t} \int_{B} k_{i j} \theta_{, i} \theta_{, j} d v d s \\
& =\int_{0}^{t} \int_{B}\left(\rho F_{i} \dot{u}_{i}+\rho q \theta\right) d v d s+\frac{1}{2} \int_{0}^{t} \int_{B}\left(\rho F_{i}(s) \dot{u}_{i}(2 t-s)-\rho F_{i}(2 t-s) \dot{u}_{i}(s)\right) d v d s \\
& \quad+\frac{1}{2} \int_{0}^{t} \int_{B}(\rho q(2 t-s) \theta(s)-\rho q(s) \theta(2 t-s)) d v d s .
\end{aligned}
$$

We now consider several estimates:

$$
\begin{aligned}
\int_{0}^{t} \int_{B}\left[\rho F_{i} \dot{u}_{i}+\rho q \theta\right] d v d s \leq & \left(\int_{0}^{t} \int_{B} \rho F_{i} F_{i} d v d s\right)^{1 / 2}\left(\int_{0}^{t} \int_{B} \rho \dot{u}_{i} \dot{u}_{i} d v d s\right)^{1 / 2} \\
& +\left(\int_{0}^{t} \int_{B} \rho q^{2} d v d s\right)^{1 / 2}\left(\int_{0}^{t} \int_{B} \rho \theta^{2} d v d s\right)^{1 / 2} \\
\leq & \left(\int_{0}^{t} \int_{B}\left[\rho F_{i} F_{i}+\rho q^{2}\right] d v d s\right)^{1 / 2}\left(\int_{0}^{t} \int_{B}\left[\rho \dot{u}_{i} \dot{u}_{i}+\rho \theta^{2}\right] d v d s\right)^{1 / 2},
\end{aligned}
$$

where we have used the inequality

$$
\sqrt{a} \sqrt{b}+\sqrt{c} \sqrt{d} \leq \sqrt{a+c} \sqrt{b+d}
$$

In a similar way, we have that

$$
\begin{aligned}
\int_{0}^{t} \int_{B}\left[\rho F_{i}(s) \dot{u}_{i}(2 t-s)-\rho F_{i}(2 t-s) \dot{u}_{i}(s)\right] d v d s \\
\leq\left(\int_{0}^{t} \int_{B} \rho F_{i} F_{i} d v d s\right)^{1 / 2}\left(\int_{t}^{2 t} \int_{B} \rho \dot{u}_{i} \dot{u}_{i} d v d s\right)^{1 / 2} \\
\quad+\left(\int_{t}^{2 t} \int_{B} \rho F_{i} F_{i} d v d s\right)^{1 / 2}\left(\int_{0}^{t} \int_{B} \rho \dot{u}_{i} \dot{u}_{i} d v d s\right)^{1 / 2} \\
\leq\left(\int_{0}^{2 t} \int_{B} \rho F_{i} F_{i} d v d s\right)^{1 / 2}\left(\int_{0}^{2 t} \int_{B} \rho \dot{u}_{i} \dot{u}_{i} d v d s\right)^{1 / 2}
\end{aligned}
$$

We can also obtain that

$$
\begin{aligned}
& \int_{0}^{t} \int_{B}[\rho q(2 t-s) \theta(s)-\rho q(s) \theta(2 t-s)] d v d s \\
& \quad \leq\left(\int_{0}^{2 t} \int_{B} \rho q^{2} d v d s\right)^{1 / 2}\left(\int_{0}^{2 t} \int_{B} \rho \theta^{2} d v d s\right)^{1 / 2} .
\end{aligned}
$$

Therefore, we see that

$$
\begin{aligned}
& \int_{B}\left(\rho \dot{u}_{i} \dot{u}_{i}+b_{i j} \alpha_{, i} \alpha_{, j}\right) d v+\int_{0}^{t} \int_{B} k_{i j} \theta_{, i} \theta_{, j} d v d s \\
& \quad \leq \frac{3}{2}\left(\int_{0}^{2 t} \int_{B}\left[\rho F_{i} F_{i}+\rho q^{2}\right] d v d s\right)^{1 / 2}\left(\int_{0}^{2 t} \int_{B}\left[\rho \dot{u}_{i} \dot{u}_{i}+\rho \theta^{2}\right] d v d s\right)^{1 / 2} .
\end{aligned}
$$


Let us assume that

$$
\sup _{t \in[0, T]} \int_{B}\left(\rho \dot{u}_{i} \dot{u}_{i}+\rho \theta^{2}\right) d v \leq N_{1}^{2}
$$

and that $t \leq T / 2$. We obtain that the inequality

$$
\begin{gathered}
\int_{B}\left(\rho \dot{u}_{i} \dot{u}_{i}+b_{i j} \alpha_{, i} \alpha_{, j}\right) d v+\int_{0}^{t} \int_{B} k_{i j} \theta_{, i} \theta_{, j} d v d s \\
\leq \frac{3}{2} T^{1 / 2} N_{1}\left[\int_{0}^{T} \int_{B}\left(\rho F_{i} F_{i}+\rho q^{2}\right) d v d s\right]^{1 / 2}
\end{gathered}
$$

is satisfied for every $t \leq T / 2$. Estimate (3.12) is fundamental in our approach. However, as we do not assume that $b_{i j}$ is a positive definite matrix, we do not have a positive quadratic form on the left side of the estimate. Thus, we will need to manipulate this term to work with a positive quadratic form. This will be the main aim of the next sections.

\section{The main result}

The aim of this section is to obtain an estimate for the solutions of the problem determined by (2.6), (2.7) and (2.8). From the inequality (3.12) and the use of the Poincaré type inequality (2.9) we will be able to get such kind of estimate.

We are going to decompose the interval $[0, T / 2]$ in a finite sequence of subintervals $I_{0}, I_{1}$, $I_{2}, \ldots, I_{m}$ such that $[0, T / 2] \subset \bigcup_{j=0}^{m} I_{j}$ and such that each intersection $I_{i} \cap I_{i+1}$ has a unique element, denoted by $\left\{t_{i}\right\}$, for $i=1, \ldots, m-1$. Then, we will obtain that

$$
\int_{B} \rho \dot{u}_{i} \dot{u}_{i} d v+\frac{1}{2} \int_{0}^{t} \int_{B} k_{i j} \theta_{, i} \theta_{, j} d v d s \leq E_{n}
$$

whenever $t \in I_{n} \cap[0, T / 2]$ where the estimate $E_{n}$ is defined by the recurrence

$$
E_{0}=\frac{3}{2} T^{1 / 2} N_{1}\left[\int_{0}^{t} \int_{B}\left(\rho F_{i} F_{i}+\rho q^{2}\right) d v d s\right]^{1 / 2}
$$

and

$$
E_{n+1}=\frac{16 C_{1} C_{2} T}{\pi} E_{n}+\frac{3}{2} T^{1 / 2} N_{1}\left[\int_{0}^{t} \int_{B}\left(\rho F_{i} F_{i}+\rho q^{2}\right) d v d s\right]^{1 / 2} .
$$

Our first step is to obtain that

$$
\int_{B} \rho \dot{u}_{i} \dot{u}_{i} d v+\frac{1}{2} \int_{0}^{t} \int_{B} k_{i j} \theta_{, i} \theta_{, j} d v d s \leq E_{0}
$$

whenever $t \in I_{0}=\left[0, \frac{\pi}{16 C_{1} C_{2}}\right]$. 
From (2.2), the arithmetic-geometric mean inequality and (2.9), we know that

$$
\begin{aligned}
& \int_{B} b_{i j} \alpha_{, i} \alpha_{, j} d v+\int_{0}^{t} \int_{B} k_{i j} \theta_{, i} \theta_{j} d v d s \\
& \quad \geq-C_{1} \int_{B} k_{i j} \alpha_{, i} \alpha_{, j} d v+\int_{0}^{t} \int_{B} k_{i j} \theta_{, i} \theta_{, j} d v d s \\
& =-2 C_{1} \int_{0}^{t} \int_{B} k_{i j} \alpha_{, i} \theta_{, j} d v d s+\int_{0}^{t} \int_{B} k_{i j} \theta_{, i} \theta_{, j} d v d s \\
& \quad \geq-2 C_{1}\left(\int_{0}^{t} \int_{B} k_{i j} \alpha_{, i} \alpha_{, j} d v d s\right)^{1 / 2}\left(\int_{0}^{t} \int_{B} k_{i j} \theta_{, i} \theta_{, j} d v d s\right)^{1 / 2}+\int_{0}^{t} \int_{B} k_{i j} \theta_{, i} \theta_{, j} d v d s \\
& \quad \geq\left(1-\frac{4 C_{1} C_{2} t}{\pi}\right) \int_{0}^{t} \int_{B} k_{i j} \theta_{, i} \theta_{, j} d v d s .
\end{aligned}
$$

If we assume that $t<\frac{\pi}{16 C_{1} C_{2}}$, we see that

$$
\int_{B} b_{i j} \alpha_{, i} \alpha_{, j} d v+\int_{0}^{t} \int_{B} k_{i j} \theta_{, i} \theta_{, j} d v d s \geq \frac{1}{2} \int_{0}^{t} \int_{B} k_{i j} \theta_{, i} \theta_{, j} d v d s
$$

Thus, in view of the estimate (3.12), we obtain the inequality (4.4).

Let us assume that we have obtained an estimate of the type

$$
\int_{B} \rho \dot{u}_{i} \dot{u}_{i} d v+\frac{1}{2} \int_{0}^{t} \int_{B} k_{i j} \theta_{, i} \theta_{, j} d v d s \leq E_{n},
$$

for $\frac{n \pi}{16 C_{1} C_{2}}<t<\frac{(n+1) \pi}{16 C_{1} C_{2}}<\frac{T}{2}$, and we want to get a similar bound for

$$
\frac{(n+1) \pi}{16 C_{1} C_{2}}<t<\min \left\{\frac{(n+2) \pi}{16 C_{1} C_{2}}, \frac{T}{2}\right\} .
$$

The analysis starts by considering the relations

$$
\begin{aligned}
& \int_{B} \rho \dot{u}_{i} \dot{u}_{i} d v+\int_{0}^{t} \int_{B} k_{i j} \theta_{, i} \theta_{, j} d v d s \\
& =\int_{B} \rho \dot{u}_{i} \dot{u}_{i} d v+\int_{B} b_{i j} \alpha_{, i} \alpha_{, j} d v+\int_{0}^{t} \int_{B} k_{i j} \theta_{, i} \theta_{, j} d v d s-\int_{B} b_{i j} \alpha_{, i} \alpha_{, j} d v \\
& \quad \leq \frac{3}{2} T^{1 / 2} N_{1}\left[\int_{0}^{T} \int_{B}\left(\rho F_{i} F_{i}+\rho q^{2}\right) d v d s\right]^{1 / 2}+C_{1} \int_{B} k_{i j} \alpha_{, i} \alpha_{, j} d v
\end{aligned}
$$

Here we have applied the estimates (2.2) and (3.12). We note that

$$
\begin{aligned}
C_{1} \int_{B} k_{i j} \alpha_{, i} \alpha_{, j} d v \leq & 2 C_{1} \int_{B} k_{i j}\left(\alpha_{, i}(t)-\alpha_{, i}\left(t_{n+1}\right)\right)\left(\alpha_{, j}(t)-\alpha_{, j}\left(t_{n+1}\right)\right) d v \\
& +2 C_{1} \int_{B} k_{i j} \alpha_{, i}\left(t_{n+1}\right) \alpha_{, j}\left(t_{n+1}\right) d v
\end{aligned}
$$


where $t_{n+1}=\frac{(n+1) \pi}{16 C_{1} C_{2}}$. We have that

$C_{1} \int_{B} k_{i j} \alpha_{, i} \alpha_{, j} d v$

$\leq 4 C_{1} \int_{t_{n+1}}^{t} \int_{B} k_{i j}\left(\alpha_{, i}(s)-\alpha_{, i}\left(t_{n+1}\right)\right) \theta_{, j} d v d s+4 C_{1} \int_{0}^{t_{n+1}} \int_{B} k_{i j} \alpha_{, i} \theta_{, j} d v d s$

$\leq 4 C_{1}\left(\int_{t_{n+1}}^{t} \int_{B} k_{i j}\left(\alpha_{, i}(s)-\alpha_{, i}\left(t_{n+1}\right)\right)\left(\alpha_{, j}(s)-\alpha_{, j}\left(t_{n+1}\right)\right) d v d s\right)^{1 / 2}\left(\int_{t_{n+1}}^{t} \int_{B} k_{i j} \theta_{, i} \theta_{, j} d v d s\right)^{1 / 2}$

$+4 C_{1}\left(\int_{0}^{t_{n+1}} \int_{B} k_{i j} \alpha_{, i} \alpha_{, j} d v d s\right)^{1 / 2}\left(\int_{0}^{t_{n+1}} \int_{B} k_{i j} \theta_{, i} \theta_{, j} d v d s\right)^{1 / 2}$

$\leq \frac{8 C_{1} C_{2}\left(t-t_{n+1}\right)}{\pi} \int_{t_{n+1}}^{t} \int_{B} k_{i j} \theta_{, i} \theta_{, j} d v d s+\frac{8 C_{1} C_{2} t_{n+1}}{\pi} \int_{0}^{t_{n+1}} \int_{B} k_{i j} \theta_{, i} \theta_{, j} d v d s$.

From (4.8) and (4.9), it follows that

$$
\begin{aligned}
& \int_{B} \rho \dot{u}_{i} \dot{u}_{i} d v+\int_{0}^{t} \int_{B} k_{i j} \theta_{, i} \theta_{, j} d v d s \\
& \leq \frac{3}{2} T^{1 / 2} N_{1}\left[\int_{0}^{T} \int_{B}\left(\rho F_{i} F_{i}+\rho q^{2}\right) d v d s\right]^{1 / 2} \\
& \quad+\frac{8 C_{1} C_{2}\left(t-t_{n+1}\right)}{\pi} \int_{0}^{t} \int_{B} k_{i j} \theta_{, i} \theta_{, j} d v d s+\frac{16 C_{1} C_{2} t_{n+1}}{\pi} E_{n} .
\end{aligned}
$$

If we assume that $t-t_{n+1}<\frac{\pi}{16 C_{1} C_{2}}$, we obtain the desired estimate (4.1)-(4.3). We note that from the recurrence (4.3), we have

$$
\begin{aligned}
E_{m} & \leq \frac{16 C_{1} C_{2} T}{\pi}\left[\frac{16 C_{1} C_{2} T}{\pi} E_{m-2}+E_{0}\right]+E_{0} \\
& \leq \cdots \leq\left(\frac{16 C_{1} C_{2} T}{\pi}\right)^{m} E_{0}+\left(\frac{16 C_{1} C_{2} T}{\pi}\right)^{m-1} E_{0}+\cdots+E_{0} .
\end{aligned}
$$

Hence,

$$
E_{m} \leq\left[\sum_{k=0}^{m}\left(\frac{16 C_{1} C_{2} T}{\pi}\right)^{k}\right] E_{0}
$$

Thus, we can conclude that the estimate

$$
\begin{aligned}
& \int_{B} \rho \dot{u}_{i} \dot{u}_{i} d v+\frac{1}{2} \int_{0}^{t} \int_{B} k_{i j} \theta_{, i} \theta_{, j} d v \\
& \quad \leq \frac{3}{2} T^{1 / 2} N_{1}\left[\sum_{k=0}^{m}\left(\frac{16 C_{1} C_{2} T}{\pi}\right)^{k}\right]\left(\int_{0}^{T} \int_{B}\left(\rho F_{i} F_{i}+\rho q^{2}\right) d v d s\right)^{1 / 2}
\end{aligned}
$$

is satisfied whenever $m$ is the first natural number such that $m>\frac{8 C_{1} C_{2} T}{\pi}$. Therefore, we have proved the following result. 
Theorem 4.1 Let $\left(u_{i}^{(1)}, \alpha^{(1)}\right)$ and $\left(u_{i}^{(2)}, \alpha^{(2)}\right)$ be the solutions of the system (1.1) corresponding to the supply terms $\left(f_{i}^{(1)}, r^{(1)}\right)$ and $\left(f_{i}^{(2)}, r^{(2)}\right)$, respectively. Then, the difference denoted by (2.5) satisfies the estimate

$$
\begin{aligned}
& \int_{B} \rho \dot{u}_{i} \dot{u}_{i} d v+\frac{1}{2} \int_{0}^{t} \int_{B} k_{i j} \theta_{, i} \theta_{, j} d v \\
& \leq \frac{3}{2} T^{1 / 2} N_{1}\left[\frac{1-\left(\frac{16 C_{1} C_{2} T}{\pi}\right)^{m+1}}{1-\frac{16 C_{1} C_{2} T}{\pi}}\right]\left(\int_{0}^{T} \int_{B}\left(\rho F_{i} F_{i}+\rho q^{2}\right) d v d s\right)^{1 / 2},
\end{aligned}
$$

where $m$ is the first natural number such that $m>8 C_{1} C_{2} T / \pi$.

\section{Continuous dependence on initial data}

The analysis proposed in Section 4 can be adapted to study the stability with respect to the initial data. Let us assume that we have two solutions $\left(u_{i}^{(1)}, \alpha^{(1)}\right)$ and $\left(u_{i}^{(2)}, \alpha^{(2)}\right)$ to the homogeneous version $\left(f_{i}=0, r=0\right)$ of the system (1.1) with the same boundary conditions, but with different initial conditions. Using the notation proposed previously, we denote by $\left(u_{i}, \alpha\right)$ the solution of the homogeneous version of the system (2.6) with homogeneous boundary conditions (2.7) and the initial conditions

$$
\begin{aligned}
& u_{i}(\boldsymbol{x}, 0)=u_{i}^{(2)}(\boldsymbol{x}, 0)-u_{i}^{(1)}(\boldsymbol{x}, 0)=u_{i}^{*}(\boldsymbol{x}), \\
& \dot{u}_{i}(\boldsymbol{x}, 0)=\dot{u}_{i}^{(2)}(\boldsymbol{x}, 0)-\dot{u}_{i}^{(1)}(\boldsymbol{x}, 0)=v_{i}^{*}(\boldsymbol{x}), \\
& \alpha(\boldsymbol{x}, 0)=\alpha^{(2)}(\boldsymbol{x}, 0)-\alpha^{(1)}(\boldsymbol{x}, 0)=\alpha^{*}(\boldsymbol{x}), \\
& \dot{\alpha}(\boldsymbol{x}, 0)=\dot{\alpha}^{(2)}(\boldsymbol{x}, 0)-\dot{\alpha}^{(1)}(\boldsymbol{x}, 0)=\theta^{*}(\boldsymbol{x}) .
\end{aligned}
$$

In this case, the energy equation gives

$$
E(t)=\int_{B}\left(\rho \dot{u}_{i} \dot{u}_{i}+c \theta^{2}+a_{i j k l} u_{i, j} u_{k, l}+b_{i j} \alpha_{, i} \alpha_{, j}\right) d v+2 \int_{0}^{t} \int_{B} k_{i j} \theta_{, i} \theta_{, j} d v d s=E(0),
$$

where

$$
E(0)=\int_{B}\left(\rho v_{i}^{*} v_{i}^{*}+c\left(\theta^{*}\right)^{2}+a_{i j k l} u_{i, j}^{*} u_{k, l}^{*}+b_{i j} \alpha_{, i}^{*} \alpha_{, j}^{*}\right) d v .
$$

As we consider the homogeneous system, the Lagrange identities argument implies that

$$
\begin{aligned}
& \int_{B}\left(\rho \dot{u}_{i} \dot{u}_{i}+b_{i j} \alpha_{, i} \alpha_{, j}-a_{i j k l} u_{i, j} u_{k, l}-c \theta^{2}\right) d v \\
& =\int_{B}\left(\rho v_{i}^{*} \dot{u}_{i}(2 t)+b_{i j} \alpha_{, i}^{*} \alpha_{, j}(2 t)-a_{i j k l} u_{i, j}^{*} u_{k, l}(2 t)-c \theta^{*} \theta(2 t)\right) d v .
\end{aligned}
$$

From (5.2) and (5.4), we obtain that

$$
\begin{aligned}
& \int_{B}\left(\rho \dot{u}_{i} \dot{u}_{i}+b_{i j} \alpha_{, i} \alpha_{, j}\right) d v+\int_{0}^{t} \int_{B} k_{i j} \theta_{, i} \theta_{, j} d v d s \\
& \quad=\frac{E(0)}{2}+\frac{1}{2} \int_{B}\left(\rho v_{i}^{*} \dot{u}_{i}(2 t)+b_{i j} \alpha_{, i}^{*} \alpha_{, j}(2 t)-a_{i j k l} u_{i, j}^{*} u_{k, l}(2 t)-c \theta^{*} \theta(2 t)\right) d v .
\end{aligned}
$$


We have that

$$
\begin{aligned}
\left|\int_{B} \rho v_{i}^{*} \dot{u}_{i}(2 t) d v\right| & \leq\left(\int_{B} \rho v_{i}^{*} v_{i}^{*} d v\right)^{1 / 2}\left(\int_{B} \rho \dot{u}_{i}(2 t) \dot{u}_{i}(2 t) d v\right)^{1 / 2} \\
\left|\int_{B} c \theta^{*} \theta(2 t) d v\right| & \leq\left(\int_{B} c\left(\theta^{*}\right)^{2} d v\right)^{1 / 2}\left(\int_{B} c \theta^{2}(2 t) d v\right)^{1 / 2} \\
\left|\int_{B} b_{i j} \alpha_{, i}^{*} \alpha_{, j}(2 t) d v\right| & \leq C_{3}\left(\int_{B} \alpha_{, i}^{*} \alpha_{, i}^{*} d v\right)^{1 / 2}\left(\int_{B} \alpha_{, j}(2 t) \alpha_{, j}(2 t) d v\right)^{1 / 2} \\
\left|\int_{B} a_{i j k h} u_{i, j}^{*} u_{k, h}(2 t) d v\right| & \leq C_{4}\left(\int_{B} u_{i, j}^{*} u_{i, j}^{*} d v\right)^{1 / 2}\left(\int_{B} u_{k, h}(2 t) u_{k, h}(2 t) d v\right)^{1 / 2} .
\end{aligned}
$$

Here, $C_{3}$ and $C_{4}$ are two calculable positive constants depending on $b_{i j}$ and $a_{i j k h}$, respectively. If we assume that

$$
\sup _{t \in[0, T]} \int_{B}\left(\rho \dot{u}_{i} \dot{u}_{i}+C_{4} u_{i, j} u_{i, j}+C_{3} \alpha_{, i} \alpha_{, i}+c \theta^{2}\right) d v<N_{2}^{2}
$$

and we take $T \geq 2 t$, we see that

$$
\int_{B}\left(\rho \dot{u}_{i} \dot{u}_{i}+b_{i j} \alpha_{, i} \alpha_{, j}\right) d v+\int_{0}^{t} \int_{B} k_{i j} \theta_{, i} \theta_{, j} d v d s \leq \frac{E(0)}{2}+\frac{1}{2} N_{2} T I^{*},
$$

where

$$
I^{*}=\left[\int_{B}\left(\rho v_{i}^{*} v_{i}^{*}+C_{3} \alpha_{, i}^{*} \alpha_{, i}^{*}+C_{4} u_{i, j}^{*} u_{i, j}^{*}+c\left|\theta^{*}\right|^{2}\right) d v\right]^{1 / 2} .
$$

Estimate (5.11) plays a similar role to the estimate (3.12) in the corresponding case.

We note that

$$
\begin{aligned}
\int_{B} b_{i j} \alpha_{, i} \alpha_{, j} d v \leq C_{1} \int_{B} k_{i j} \alpha_{, i} \alpha_{, j} d v & \leq 2 C_{1} \int_{B} k_{i j}\left(\alpha_{, i}-\alpha_{, i}^{*}\right)\left(\alpha_{, j}-\alpha_{, j}^{*}\right) d v+2 C_{1} \int_{B} k_{i j} \alpha_{, i}^{*} \alpha_{, j}^{*} d v \\
& \leq 4 C_{1} \int_{0}^{t} \int_{B} k_{i j}\left(\alpha_{, j}-\alpha_{, j}^{*}\right) \theta_{, j} d v d s+2 C_{1} \int_{B} k_{i j} \alpha_{, i}^{*} \alpha_{, j}^{*} d v \\
& \leq \frac{8 C_{1} C_{2} t}{\pi} \int_{0}^{t} \int_{B} k_{i j} \theta_{, i} \theta_{, j} d v d s+2 C_{1} \int_{B} k_{i j} \alpha_{, i}^{*} \alpha_{, j}^{*} d v .
\end{aligned}
$$

We can determine a recurrence similar to (4.2) and (4.3). We define $H_{n}$ by means of the recurrence

$$
H_{0}=\frac{E(0)}{2}+2 C_{1} \int_{B} k_{i j} \alpha_{, i}^{*} \alpha_{, j}^{*} d v+\frac{1}{2} N_{2} T I^{*}
$$

and

$$
H_{n+1}=\frac{E(0)}{2}+\frac{1}{2} N_{2} T I^{*}+\frac{16 C_{1} C_{2} T}{\pi} H_{n}
$$

If we consider the estimate (5.13) and we choose $t_{1}=\frac{\pi}{16 C_{1} C_{2}}$, we see that the estimate

$$
\int_{B} \rho \dot{u}_{i} \dot{u}_{i} d v+\frac{1}{2} \int_{0}^{t} \int_{B} k_{i j} \theta_{, i} \theta_{, j} d v d s \leq H_{0}
$$


is satisfied whenever $0<t<t_{1}<T / 2$.

Let us assume that the estimate

$$
\int_{B} \rho \dot{u}_{i} \dot{u}_{i} d v+\frac{1}{2} \int_{0}^{t} \int_{B} k_{i j} \theta_{, i} \theta_{, j} d v d s \leq H_{n}
$$

holds whenever $\frac{n \pi}{16 C_{1} C_{2}}<t \leq \frac{(n+1) \pi}{16 C_{1} C_{2}}<\frac{T}{2}$. We want to obtain a similar estimate in the interval

$$
\frac{(n+1) \pi}{16 C_{1} C_{2}}<t<\min \left\{\frac{(n+2) \pi}{16 C_{1} C_{2}}, \frac{T}{2}\right\} \text {. }
$$

As in (4.8), it turns out

$$
\begin{aligned}
& \int_{B} \rho \dot{u}_{i} \dot{u}_{i} d v+\int_{0}^{t} \int_{B} k_{i j} \theta_{, i} \theta_{, j} d v d s \\
& \leq \frac{E(0)}{2}+\frac{1}{2} N_{2} T I^{*}+2 C_{1} \int_{B} k_{i j}\left(\alpha_{, i}(t)-\alpha_{, i}\left(t_{n+1}\right)\right)\left(\alpha_{, j}(t)-\alpha_{, j}\left(t_{n+1}\right)\right) d v \\
& \quad+2 C_{1} \int_{B} k_{i j} \alpha_{, i}\left(t_{n+1}\right) \alpha_{, j}\left(t_{n+1}\right) d v .
\end{aligned}
$$

Moreover,

$$
\begin{aligned}
\int_{B} & k_{i j}\left(\alpha_{, i}(t)-\alpha_{, i}\left(t_{n+1}\right)\right)\left(\alpha_{, j}(t)-\alpha_{, j}\left(t_{n+1}\right)\right) d v \\
& =2 \int_{t_{n+1}}^{t} \int_{B} k_{i j}\left(\alpha_{, i}(s)-\alpha_{, i}\left(t_{n+1}\right)\right) \theta_{, j}(s) d v d s \\
& \leq 2\left(\int_{t_{n+1}}^{t} \int_{B} k_{i j}\left(\alpha_{, i}(s)-\alpha_{, i}\left(t_{n+1}\right)\right)\left(\alpha_{, j}(s)-\alpha_{, j}\left(t_{n+1}\right)\right) d v d s\right)^{1 / 2}\left(\int_{t_{n+1}}^{t} \int_{B} k_{i j} \theta_{, i} \theta_{, j} d v d s\right)^{1 / 2} \\
& \leq \frac{4 C_{2}\left(t_{n}-t_{n+1}\right)}{\pi} \int_{0}^{t} \int_{B} k_{i j} \theta_{, i} \theta_{, j} d v d s
\end{aligned}
$$

and

$$
\int_{B} k_{i j} \alpha_{, i}\left(t_{n+1}\right) \alpha_{, j}\left(t_{n+1}\right) d v \leq \frac{2 C_{2} t_{n+1}}{\pi} \int_{B} k_{i j} \theta_{, i}\left(t_{n+1}\right) \theta_{, j}\left(t_{n+1}\right) d s \leq \frac{4 C_{2} t_{n+1}}{\pi} H_{n} .
$$

From (5.18)-(5.20), it follows that

$$
\begin{aligned}
& \int_{B} \rho \dot{u}_{i} \dot{u}_{i} d v+\int_{0}^{t} \int_{B} k_{i j} \theta_{, i} \theta_{, j} d v d s \\
& \quad \leq \frac{E(0)}{2}+\frac{1}{2} N_{2} T I^{*}+\frac{8 C_{1} C_{2}\left(t_{n}-t_{n+1}\right)}{\pi} \int_{0}^{t} \int_{B} k_{i j} \theta_{, i} \theta_{, j} d v d s+\frac{16 C_{1} C_{2} t_{n+1}}{\pi} H_{n} .
\end{aligned}
$$

So, we get that

$$
\int_{B} \rho \dot{u}_{i} \dot{u}_{i} d v+\frac{1}{2} \int_{0}^{t} \int_{B} k_{i j} \theta_{, i} \theta_{, j} d v d s \leq \frac{E(0)}{2}+\frac{1}{2} N_{2} T I^{*}+\frac{16 C_{1} C_{2} T}{\pi} H_{n}
$$


which is the desired estimate. In a similar way to the argument proposed at the end of Section 4 , we can obtain the estimate

$$
\begin{aligned}
& \int_{B} \rho \dot{u}_{i} \dot{u}_{i} d v+\frac{1}{2} \int_{0}^{t} \int_{B} k_{i j} \theta_{, i} \theta_{, j} d v d s \\
& \leq\left[\frac{1-\left(\frac{16 C_{1} C_{2} T}{\pi}\right)^{m+1}}{1-\frac{16 C_{1} C_{2} T}{\pi}}\right]\left(\frac{E(0)}{2}+2 C_{1} \int_{B} k_{i j} \alpha_{, i}^{*} \alpha_{, j}^{*} d v+\frac{1}{2} N_{2} T I^{*}\right),
\end{aligned}
$$

where $m$ is the first natural number such that $m>\frac{8 C_{1} C_{2} T}{\pi}$. Thus, we conclude the Hölder stability with respect to the initial data.

\section{References}

[1] K.A. Ames, L.E. Payne, Continuous dependence on initial-time geometry for a thermoelastic system with sign-indefinite elasticities, J. Math. Anal. Appl. 189 (1995) 693-714.

[2] K.A. Ames, B. Straughan, Continuous dependence results for initially prestressed thermoelastic bodies, Int. Jour. Eng. Sci. 30 (1992) 7-13.

[3] V.A. Cimmelli, F. dell'Isola, A moving boundary problem describing the growth of a droplet in its vapour, Arch. Mech. 45 (1993) 625-634.

[4] F. dell'Isola, Linear growth of a liquid droplet divided from its vapour by a "soap bubblelike fluid interface, Int. Jour. Eng. Sci. 27 (1989) 1053-1067.

[5] A.E. Green, P.M. Naghdi, On undamped heat waves in an elastic solid, J. Thermal Stresses 15 (1992) 253-264.

[6] A.E. Green, P.M. Naghdi, Thermoelasticity without energy dissipation, J. Elasticity 31 (1993) 189-208.

[7] A.E. Green, P.M. Naghdi, A unified procedure for construction of theories of deformable media, I. Classical continuum physics, II. Generalized continua, III. Mixtures of interacting continua, Proc. Roy. Soc. London A 448 (1995) 335-356, 357-377, 379-388.

[8] D. Iesan, On the theory of thermoelasticity without energy dissipation, J. Thermal Stresses 21 (1998) 295-307.

[9] D. Iesan, Thermopiezoelectricity without energy dissipation, Proc. Roy. Soc. London A 464 (2008) 631-656.

[10] D. Iesan, R. Quintanilla, On the thermoelastic bodies with inner structure and microtemperatures, J. Math. Anal. Appl. 354 (2009) 12-23.

[11] F. John, Continuous dependence on data for solutions of partial differential equations with a prescribed bound, Commun. Pure Appl. Math. 13 (1960) 551-585. 
[12] R. J. Knops, L. E. Payne, Improved estimates for continuous data dependence in linear elastodynamics, Math. Proc. Cambr. Philosoph. Soc. 103 (1988) 535-559.

[13] B. Lazzari, R. Nibbi, On the exponential decay in thermoelasticity without energy dissipation and of type III in presence of an absorbing boundary, J. Math. Anal. Appl. 338 (2008) 317-329.

[14] M.C. Leseduarte, A. Magaña, R. Quintanilla, On the time decay of solutions in porousthermo-elasticity of type II, Discrete and Continuous Dynamical Systems, Series B 13 (2010) 375-391.

[15] M.C. Leseduarte, R. Quintanilla, On uniqueness and continuous dependence in type III thermoelasticity, J. Math. Anal. Appl. 395 (2012) 429-436.

[16] Z. Liu, R. Quintanilla, Analiticity of solutions in the type III thermoelastic plates, IMA Journal of Applied Mathematics 75 (2010) 356-365.

[17] Z. Liu, R. Quintanilla, Energy decay rates of mixed type II and type III thermoelastic system, Discrete and Continuous Dynamical Systems, Series B 14 (2010) 1433-1444.

[18] Y. Liu, C. Lin, Phragmén-Lindelöf alternative and continuous dependence-type results for the thermoelasticity of type III, Applicable Analysis: An Inernational Journal 83 (2008) 431-449.

[19] S.A. Messaoudi, A. Soufyane, Boundary stabilization of memory type in thermoelasticity of type III, Appl. Anal. 87 (2008) 13-28.

[20] P. Puri, P.M. Jordan, On the propagation of plane waves in type-III thermoelastic media, Proc. Roy. Soc. London A 460 (2004) 3203-3221.

[21] Y. Qin, S. Deng, L. Huang, Z. Ma, X. Su, Global existence for the three-dimensional thermoelastic equations of Type II, Quart. Appl. Math. 68 (2010) 333-348.

[22] R. Quintanilla, On the spatial behaviour in thermoelasticity without energy dissipation, J. Thermal Stresses 21 (1999) 213-224.

[23] R. Quintanilla, Damping of end effects in a thermoelastic theory, Appl. Math. Letters 14 (2001) 137-141.

[24] R. Quintanilla, Structural stability and continuous dependence of solutions of thermoelasticity of type III, Discrete Continuous Dynamical Systems-Series B 1 (2001) 463-470.

[25] R. Quintanilla, Some Remarks on Growth and Uniqueness in Thermoelasticity, IJMMS vol. 2003, 10 (2003) 617-623.

[26] R. Quintanilla, Convergence and structural stability in thermoelasticity, Appl. Math. Comp. 135 (2003) 287-300.

[27] R. Quintanilla, Impossibility of localization in linear thermoelasticity, Proc. Roy. Soc. London A 463 (2007) 3311-3322. 
[28] R. Quintanilla, R. Racke, Stability in thermoelasticity of type III, Discrete and Continuous Dynamical Systems, Series B 3 (2003) 383-400.

[29] R. Quintanilla, B. Straughan, Growth and uniqueness in thermoelasticity, Proc. Roy. Soc. London A 456 (2000) 1419-1429.

[30] R. Quintanilla, B. Straughan, A note on discontinuity waves in type III thermoelasticity, Proc. Roy. Soc. London A 460 (2004) 1169-1175.

[31] R. Quintanilla, B. Straughan, Energy bounds for some non-standard problems in thermoelasticity, Proc. Roy. Soc. London A 461 (2005) 1147-1162.

[32] S. Rionero, S. Chirita, The Lagrange identity method in linear thermoelasticity, Int. Jour. Eng. Sci. 25 (1987) 935-947.

[33] N.S. Wilkes, Continuous dependence and instability in linear thermoelasticity, SIAM J. Math. Anal. 11 (1980) 292-299.

[34] L. Yang, Y.G. Wang, Well-posedness and decay estimates for Cauchy problems of linear thermoelastic systems of type III in 3-D, Indiana Univ. Math. J. 55 (2006) 1333-1361. 